GRADIATION\&APPLICATIONS

ISSN 2466-4294 (online) | rad-journal.org

Vol. 2 | Issue 1 | pp. $58-61$

doi: 10.21175/RadJ.2017.01.012

Original research paper

\title{
REFRACTIVE PROPERTIES OF THE BIO- AND NANO-STRUCTURED MATERIALS AS AN INDICATOR OF THE MODEL MATRIX MACRO PARAMETER MODIFICATION*
}

\author{
Natalia Kamanina ${ }^{1,2^{* *}}$ \\ ${ }^{1}$ Lab for Photophysics of Media with Nanoobjects, Vavilov State Optical Institute, St.-Petersburg, Russia \\ ${ }^{2}$ Saint-Petersburg Electrotechnical University (“LETI”), St. Petersburg, Russia
}

\begin{abstract}
In the current paper, a unique role of the photorefractive features of the nano- and bio -doped organic systems has been considered as an indicator of the change of dynamic, structural, spectral and conductive characteristics. The paper presents some innovative views about the tendency towards the concurrent role of the bioobject sensitization of the organic matrixes in comparison with the nano-object doping one. The results have been supported both by the experimental four-wave mixing technique data as well as by the qualitative model.
\end{abstract}

Key words: Laser-matter interaction, refractive properties, organics, nano- and bio-structuring, complex formation

\section{INTRODUCTION}

Because of the relevance of the physical and chemical effects study in the new structured organic materials, including liquid crystal ones, investigation of the properties of innovative structured organic materials with new nano- and bio-objects is actual, modern and quite popular [1]-[5]. Organic systems are able to compete in their structural and optical properties with the bulk inorganic classical materials, remembering the ability to use the structured organic systems to optimize the technological processes for nano- and bio-technologies. Moreover, organic structured thin-film structures can be successfully use for the purposes of telecommunications, display, laser, biomedical engineering, microscopy techniques, industrial production, etc.

It should be mentioned that the study of the refractive effects in organic materials has a special place in the development of modern innovative technologies. This is due to the substantial correlation between refractive and photoconductive features. Really, when one applies the electric field of the laser wave to treat the matter, it should be remembered that the electric field of the laser wave is larger than the intra-atomic electric field $E_{a}$, correlated with the electron charge $e$ and with the Bohr radius $a$. Thus, we should draw the attention on the nonlinear optical photorefractive features. The photon energy lies in the range of the electronic and vibrational transitions in matter, therefore, this circumstance allows the use of light and, more particularly laser radiation, to obtain unique information about dipole moment, absorption cross section, charge carrier mobility, order parameters, etc. characteristics of the matter. The use of the laser sources provokes the drastic basic properties modification of the investigated matrix systems with good advantage.

This report summarized the prospects of the unique role of the refractive characteristics of the model organic systems which reveals other macro parameters change.

\section{EXPERIMENTAL CONDITIONS}

It should be mentioned that to study the refractive coefficients the scientists have generally used three schemes: $Z$-scanning, third harmonic generation and four-wave mixing technique. The last one have some advantage due to reversible process activated in the two modes: Bragg- and Raman-Nath conditions. The Raman-Nath diffraction mode has been made when the spatial frequency $\Lambda$ and the thickness $d$ of the structure is coincided with the relation: $\Lambda^{-1} \geq d$. Using four-wave mixing of laser beams (see Fig.1) in the nanosecond range of durations (10-20 ns), at the wavelength of the second harmonic of a neodymium laser $(\lambda=532 \mathrm{~nm})$, with variation, as energy density $W$ or spatial frequency $\Lambda$, the investigation of the change of refractive properties of a large group of conjugated organic materials by reversible recording of the diffraction grating in the first order of the diffraction have been made.

\footnotetext{
* The paper was presented at the Fourth International Conference on Radiation and Applications in Various Fields of Research (RAD 2016), Niš, Serbia, 2016.

nvkamanina@mail.ru
} 
N. Kamanina, Refractive Properties of the Bio- and Nano-Structured Materials..., Rad. Applic., 2017, 2, 1, 58-61

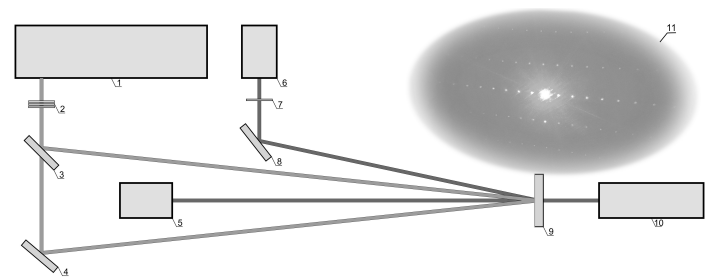

Figure 1. Experimental setup: $1-$ Nd-laser with the second harmonic convertor, 2,7 - filters, 3,4,8 - mirrors, 5,6 - photo detectors, 9 - samples, 10 - additional Nd- or He-Ne-laser for readout, 11 -diffraction orders

As the nano- and bio-sensitizers the fullerenes, shungites, carbon nanotubes (NT), quantum dots (CT), oxides graphene, DNA have been used. As the model organic matrixes, the polyimide (PI) with triphenylamine donor fragment, 2-cyclooctylamino-5nitropyridine (COANP), and nematic liquid crystal (LC) from the cyanobiphenyl group have been tested. Nano-objects-dopant concentration $C$ has been varied in the range of 0.003-1.0 wt.\%; the ones of carbon nanotubes has been in the range of 0.01-0.1 wt.\%; bioobjects dopant content (red fish DNA) has been $\sim 4.72$

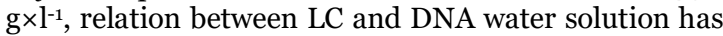
been $\sim 5: 1$.

The films have been developed using centrifuge deposition. The thickness of the films was 2-5 micrometers. The LC cell thickness was 5-10 micrometers. The nanostructured LC films have been placed onto glass substrates covered with transparent conducting layers based on ITO contacts.

\section{RESULTS AND DisCUSSION}

The values of the light-induced additives $\Delta n_{\text {i }}$ to the refractive index $n$ have been calculated from the diffraction efficiency $\eta$ via the approach shown in the papers [6],[7]. This approach has been extended for the estimation of the refractive parameters of the nanoand bio-structured organics in the papers [8]-[12]. Using Raman-Nath diffraction conditions the laserinduced change of the refractive index can be estimated as following:

$$
\eta=\frac{I_{1}}{I_{0}}=\left(\frac{\pi \Delta n_{i} \mathrm{~d}}{2 \lambda}\right)^{2}
$$

The cubic nonlinearity $\chi^{(3)}$ and the nonlinear refraction $n_{2}$ have been defined via equations:

$$
\begin{aligned}
& n=n_{0}+n_{2} I \\
& n_{2}=\frac{16 \pi^{2}}{n_{0} c} \chi^{(3)}
\end{aligned}
$$

Here $\Delta n_{\mathrm{i}}$ - laser-induced change of the refractive index, $I_{1}$ is the intensity in the first diffraction order, $I_{0}$ is the input laser intensity, $d$ is the thickness of the medium, $\lambda$ is the wavelength of the light incident on the medium, $c$ is the light velocity.
It is established that the refractive parameters, namely $n_{2}$ and $\chi^{(3)}$ are comparable with those obtained for three-dimensional inorganic silicon structures. But, it should be noticed that the technology of obtaining the thin films of conjugated organic materials is significantly easier, and the thickness is an order of magnitude less. The main results of the largest lightinduced change of the refractive index $\Delta n_{\mathrm{i}}$ in a number of structured organic model materials (for example polyimide PI, COANP and nematic LCs one) with different concentrations of the sensitizers are shown in Table 1.

The explanation of the organic conjugated systems refractive properties change when their volume has been structured is given in the framework of the significant growth of the polarizability. It is connected with the fact that the dipole moment and the way of the transfer of charge carriers from intramolecular electron donor is not on the intramolecular acceptor fragment but to the intermolecular acceptor with high electron affinity. Moreover, intermolecular acceptor delocalizes of not one, but a larger number of carriers [8]-[12]. Qualitative picture is shown in Fig.2, left part. Refractive grating recorded under the conditions when reversible regime has been transferred to the irreversible one is shown in Fig.2, right part. Analyzing the process presented in Fig.2 one can testify that in the conjugated organics with the initial D-A interaction the electron prefers to move to the nano- or bio-objects as the effective intermolecular electron acceptor with the high value of the electron affinity energy. For example, the electron affinity energy of the polyimide intramolecular acceptor fragment is close to 1.1-1.4 eV, the electron affinity energy of shungite structure is $\sim 2$ $\mathrm{eV}$, the one for fullerenes is $\sim 2.65 \mathrm{eV}$, the one for quantum dots is placed in the range of $3.8-4.2 \mathrm{eV}$. Thus, the intermolecular acceptor fragment electron affinity energy is essentially larger. It provokes the change of the electron density in the media. Moreover, the electron pathway is also longer for the intermolecular process than for the intramolecular one. That reveals the clearly larger dipole moment.

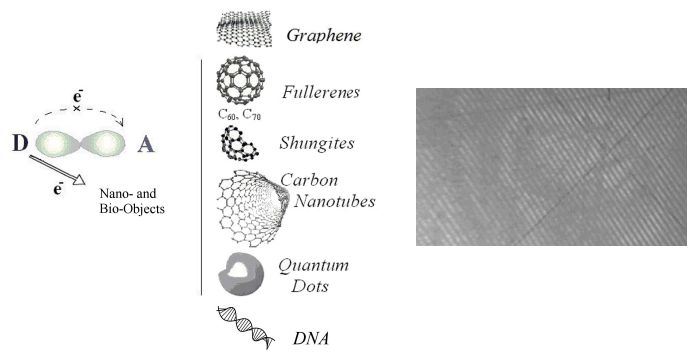

Figure 2. Qualitative model of the electrons transfer from the intramolecular donor fragment to the intermolecular acceptor

It should be said that the calculated values of nonlinear refraction $n_{2}$ and third order nonlinear susceptibility $\chi^{(3)}$ are included in a range of values: $n_{2}=10^{-10}-10^{-9} \mathrm{~cm}^{2} \times \mathrm{W}^{-1}$ and $\chi^{(3)}=10^{-10}-10^{-9} \mathrm{~cm}^{3} \times \mathrm{erg}^{-1}$. 
N. Kamanina, Refractive Properties of the Bio- and Nano-Structured Materials..., Rad. Applic., 2017, 2, 1, 58-61

Table 1. Induced change of the refractive index

\begin{tabular}{|c|c|c|c|c|}
\hline Materials & $\begin{array}{c}C, \\
\text { wt.\% }\end{array}$ & $\begin{array}{c}W, \\
\mathrm{~J} \times \mathrm{cm}^{-2}\end{array}$ & $\begin{array}{c}\Lambda, \\
\mathrm{mm}^{-1}\end{array}$ & $\Delta n_{\mathrm{i}}$ \\
\hline Pure PI & o & 0.6 & 90 & $10^{-4}-10^{-5}$ \\
\hline $\begin{array}{c}\text { PI+QD } \\
\text { CdSe(ZnS) }\end{array}$ & 0.003 & $0.2-0.3$ & $\begin{array}{l}90- \\
100\end{array}$ & $2.0 \times 10^{-3}$ \\
\hline PI+shungite & 0.2 & $\begin{array}{c}0.063^{-} \\
0.1 \\
\end{array}$ & 150 & $3.8-5.3 \times 10^{-3}$ \\
\hline $\begin{array}{l}\text { PI+graphene } \\
\text { oxide }\end{array}$ & 0.1 & 0.2 & 100 & $3.4 \times 10^{-3}$ \\
\hline $\mathrm{PI}+\mathrm{C}_{60}$ & 0.2 & $0.5-0.6$ & 90 & $4.2 \times 10^{-3}$ \\
\hline $\mathrm{PI}+\mathrm{C}_{70}$ & 0.2 & 0.6 & 90 & $4.68 \times 10^{-3}$ \\
\hline $\mathrm{PI}+\mathrm{CNTs}$ & 0.1 & $0.5-0.8$ & 90 & $5.7 \times 10^{-3}$ \\
\hline $\mathrm{PI}+\mathrm{CNTs}$ & 0.05 & 0.3 & 150 & $4.5 \times 10^{-3}$ \\
\hline $\mathrm{PI}+\mathrm{CNTs}$ & 0.07 & 0.3 & 150 & $5.0 \times 10^{-3}$ \\
\hline $\mathrm{PI}+\mathrm{CNTs}$ & 0.1 & 0.3 & 150 & $5.5 \times 10^{-3}$ \\
\hline PI+DWCNTs & 0.1 & $\begin{array}{c}0.063^{-} \\
0.1\end{array}$ & 100 & $9.4 \times 10^{-3}$ \\
\hline PI+DWCNTs & 0.1 & $\begin{array}{c}0.063^{-} \\
0.1\end{array}$ & 150 & $7.0 \times 10^{-3}$ \\
\hline $\begin{array}{c}\mathrm{LC}+\mathrm{COANP} \\
-\mathrm{C}_{70} \\
\end{array}$ & 1 & $30 \times 10^{-3}$ & 90 & $1.45^{\times 10^{-3}}$ \\
\hline $\begin{array}{c}\mathrm{LC}+\mathrm{COANP} \\
-\mathrm{C}_{70} \\
\end{array}$ & 1 & $30 \times 10^{-3}$ & 130 & $1.1 \times 10^{-3}$ \\
\hline $\begin{array}{c}\text { LC+COANP } \\
- \text { CNTs }\end{array}$ & 0.5 & $18 \times 10^{-3}$ & $\begin{array}{l}90- \\
100 \\
\end{array}$ & $3.2 \times 10^{-3}$ \\
\hline $\mathrm{LC}+\mathrm{DNA}$ & 0.1 & 0.1 & $\begin{array}{l}90- \\
120\end{array}$ & $1.39 \times 10^{-3}$ \\
\hline $\begin{array}{c}\mathrm{LC}+\mathrm{QD} \\
\mathrm{CdSe}(\mathrm{ZnS})+ \\
\text { DNA } \\
\end{array}$ & 0.1 & 0.1 & $\begin{array}{l}90- \\
120\end{array}$ & $1.35^{\times 10^{-3}}$ \\
\hline $\begin{array}{c}\mathrm{LC}+\mathrm{QD} \\
\mathrm{CdSe}(\mathrm{ZnS})+ \\
\text { DNA } \\
\end{array}$ & 0.1 & 0.1 & 130 & $1.0 \times 10^{-3}$ \\
\hline Pure LC & 0 & \multicolumn{2}{|c|}{$\begin{array}{l}0.2 \mathrm{~W} \times \mathrm{cm}^{-2} \text { at } \\
514.5 \mathrm{~nm}[13]\end{array}$} & $0.16 \times 10^{-3}$ \\
\hline
\end{tabular}

The growth of the refractive coefficients is coincided with the increase of the absorption cross section (see Ref.14), it is correlated with the change of the photoconductive properties (charge carriers mobility increase due to an increase of the length of the barrier free electron pathway). Moreover, it is revealed the changes of the order parameter of the studied materials (see. Ref.15). Furthermore, the infrared shift in their spectral characteristics has been detected (see Ref.16 and Ref.17). On the one hand, these correlations can be used for the extended applying of organic conjugated structured materials in industry and biomedicine; on the other hand, it can provoke the ability of the competing influence of bio-objects, in comparison with nano-objects, due to nontoxicity and easy renewability of the biological structures from World Ocean.

It should be mentioned that the classical ellipsometry method has been used as an additional one in order to support the increase of the refractive parameters. Really, the data shown in Fig.3 support this fact. The experiments have been performed using Horiba Jobin Yvon Uvisel device with the PSA-scheme. One can see from the Fig.3 that an introduction of the nano- and bio-particles provokes the increase of the refractive index in comparison with the same value regarded to the pure LC. In the current experiments the intermolecular charge transfer complex based on the COANP-C $\mathrm{C}_{70}$ has been used as well as the DNA molecules as the bio-sensitizer have been applied. The nano- and bio-structuration process leads to activate the self-assembly mechanism and permits to observe the new large oriented numerous domains in the doped LC media. This evidence is connected with the nanoobjects-doped organics order parameters increase which has been previously observed in the Ref.15.

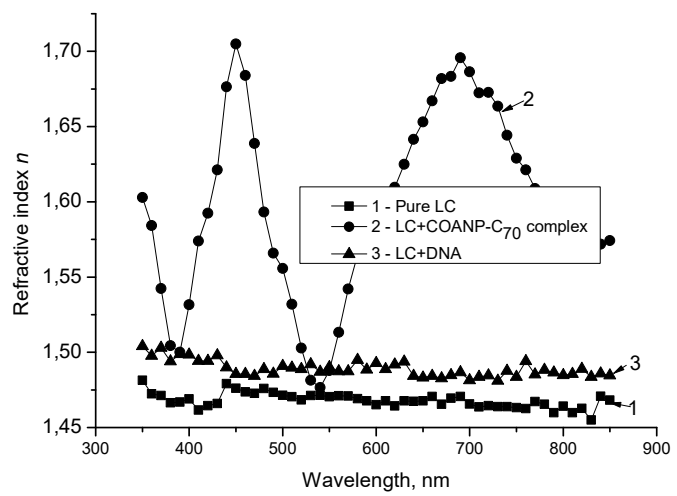

Figure 3. Spectral dependence of the refractive index $n$ via the nano- and bio-structuration of the LC mesophase

\section{CONCLUSION}

It should be remarked, that at present time different scientific groups have studied the organic photorefractive materials and have found the extended area of their application in the technique and biomedicine [18],[19]. But, it should be mentioned, that basically the authors have drawn the attention on the structuring process influence on the volume of the organics. But, for the current paper author opinion it is equally important to consider the volume properties of the organics and their interface via structuring both of ones. The evidence of the dramatic effect of the interface on the spectral and photorefractive features of the liquid crystal thin films doped with lanthanides nanoparticles have been revealed in the paper [20].

To summarize the results of the current paper, one can testify that the nano- and bio-structuring of the organics provokes the dramatic change of the refractive features. The refractive parameters of the organic thin film structures can be compared with the ones obtained for the bulk silicon materials. The refractive characteristics change can be considered as the indicator of the other important materials macro parameters modification. Photoconductive and dynamic properties as well as the structural and spectral ones can be changed too. As dominant role in this process the increase of the local volume polarizability, namely $\chi^{(3)}$ has been shown. It is proportional to the dipole polarizability. Thus, from the one side, it is correlated with an increase of the absorption cross section, and from the other side it is connected with the charge carrier mobility due to the reason that the barrier free pathway for the electrons 
N. Kamanina, Refractive Properties of the Bio- and Nano-Structured Materials..., Rad. Applic., 2017, 2, 1, 58-61

can be dramatically increases via intermolecular charge transfer. The nano- and bio-objects structuration leads to find the similar values of the refractive parameters. Thus, bio-objects doping sometimes can compete with the nano-doping one.

Finally, it should be mentioned that future experimental evidences of the intermolecular charge transfer complex should be supported via electron microscopy. Moreover, the organic thin films surface parameters (roughness, etc.) and their influence on the refractive and conductive features should be considered too.

Acknowledgement: The paper is a part of the research done within the RFBR Grants No. 1O-O3o0916 (2010-2012) and No.13-O3-00044 (2013-2015) as well as it supported by the FP7 Program, Marie Curie International Researchers Exchange Proposal "BIOMOLEC" (2011-2015).

\section{REFERENCES}

1. L. W. Tutt, T. F. Boggess, "A review of optical limiting mechanisms and devices using organics, fullerenes, semiconductors and other materials," Progress in Quantum Electronics, vol. 17, no. 4, pp. 299-338, 1993.

2. I. C. Khoo, "Holographic grating formation in dye- and fullerene $\mathrm{C}_{60}$-doped nematic liquid-crystal film," Optics Letters, vol. 20, no. 20, pp. 2137-2139, 1995.

3. H. Ono, N. Kawatsuki, "Orientational photorefractive gratings observed in polymer dispersed liquid crystals doped with fullerene," Jap. J. Appl. Phys., Part 1., vol. 36 , no. 10 , pp. 6444-6448, 1997 .

4. L. Lucchetti, F. Simoni, "Coarsening and phase separation in ultraviolet cured polymer dispersed liquid crystals," J. Appl. Phys., vol. 88, no.7, pp. 3934-3940, 2000.

5. I. C. Khoo, Y. Z. Williams, B. Lewis, T. Mallouk, "Photorefractive CdSe and Gold Nanowire-Doped Liquid Crystals and Polymer-Dispersed-Liquid-Crystal Photonic Crystals," Mol. Cryst. Liq. Cryst., vol. 446, pp. $233-44,2006$.

6. S. A. Akhmanov, and S. Yu. Nikitin, Physical Optics, Moscow University Press: Moscow, [in Russia, 19986 656 p.]; S. A. Akhmanov and S. Yu. Nikitin,). Physical Optics, Oxford University Press: USA, 1997.

7. R. J. Collier, C. B. Burckhardt, L. H. Lin, "Optical Holography”, Academic Press: New York and London. 1971.

8. N. V. Kamanina, "Fullerene-dispersed liquid crystal structure: dynamic characteristics and self-organization processes," Physics-Uspekhi, vol. 48, no. 4, pp. 419-427, 2005.
9. N. V. Kamanina, A. Emandi, F. Kajzar, A.-J. Attias, Mol. Cryst. Liq. Cryst., vol. 486, pp.1-11, 2008.

10. N. V. Kamanina, "The effect of the charge transfer pathway during intermolecular complex formation on nonlinear optical and photoconducting properties of nanocomposites," Technical Physics Letters, vol. 38, no. 2, pp. 114-117, 2012.

11. N. V. Kamanina, S. V. Serov, N. A. Shurpo, S. V. Likhomanova, D. N. Timonin, P. V. Kuzhakov, N. N. Rozhkova, I. V. Kityk, K. J. Plucinski, D. P. Uskokovic, $J$ Mater Sci: Mater Electron, published on-line 26 January 2012, August 2012, vol. 23, no. 8, pp. 15381542, 2012.

12. N. V. Kamanina, S. V. Serov, Y. Bretonniere, and C. Andraud, Journal of Nanomaterials, Hindawi Publishing Corporation, Article ID 278902, Volume 2015 (2015), 5 pages DOI: $10.1155 / 2015 / 278902$

13. I. C. Khoo, H. Li, Y. Liang, "Observation of orientation photorefractive effects in nematic liquid crystals," Opt. Lett., vol.19, no.21, pp.1723-1725, 1994.

14. N. V. Kamanina, "Mechanisms of optical limiting in $\pi$ conjugated organic system: fullerene-doped polyimide," Synthetic Metals, vol. 127, nos. 1-3, pp. 121-128, 2002.

15. N. V. Kamanina, A. V. Komolkin, N. P. Yevlampieva, "Variation of the orientational order parameter in a nematic liquid Crystal-COANP-C $\mathrm{C}_{70}$ composite structure," Tech. Phys. Lett., vol. 31, no. 6, pp.478-48o, 2005.

16. A. V. Varnaev, A. P. Zhevlakov, and N. V. Kamanina, "On the possibility of writing thin amplitude-phase holograms in the near infrared range $(\lambda=1315 \mathrm{~nm})$ using polyimide-based fullerene-containing conjugated organic structures," Optics and Spectroscopy, vol. 93, no. 3, pp. 327-329, 2002

17. N. V. Kamanina and A. I. Plekhanov, "Mechanisms of optical limiting in fullerene-doped $\pi$-conjugated organic structures demonstrated with polyimide and COANP molecules," Optics and Spectroscopy, vol. 93, no. 3, pp. 408-415, 2002.

18. S. Köber, M. Salvador and K. Meerholz, "Organic photorefractive Materials and Applications", Advanced Materials, vol. 23, no. 41, pp. 4725-4763, 2011.

19. Y. Li, Ka-Di Zhu, "Tunable optical Kerr effects of DNAs coupled to quantum dots," Nanoscale Research Letters, vol. 7, 6 pages, 2012.

Retrieved from: http://www.nanoscalereslett.com/content/7/1/660

20. N. V. Kamanina, Yu. A. Zubtcova, A. A. Kukharchik, C. Lazar, and I. Rau, "Control of the IR-spectral shift via modification of the surface relief between the liquid crystal matrixes doped with the lanthanide nanoparticles and the solid substrate," Optics Express, vol. 24, no. 2, 6 pages, 2016. 